\title{
Changes in the hemolytic activity of Candida species by common electrolytes
}

\author{
Lei Wan ${ }^{1,2+}$, Gang Luo ${ }^{3+}$, Haibin Lu ${ }^{2}$, Dongying Xuan ${ }^{2}$, Hong Cao ${ }^{1 *}$ and Jincai Zhang ${ }^{2 *}$
}

\begin{abstract}
Background: Hemolysins are crucial virulence factors which help pathogens to survive and persist in the host. This study investigated whether common electrolytes will affect the hemolysins of Candida species. The hemolysins from 25 Candida isolates were investigated using a plate specially designed for Candida species in the presence of three electrolytes, $\mathrm{CaCl}_{2}, \mathrm{NaCl}$ and $\mathrm{KCl}$, at different concentrations. The hemolytic activity was determined after $48 \mathrm{~h}$ and the hemolytic index was calculated.
\end{abstract}

Results: All three electrolytes caused a decrease in the hemolytic activity. Significant differences existed between varying concentrations of $\mathrm{NaCl}$, while no significant differences existed for the $\mathrm{CaCl}_{2}$ and $\mathrm{KCl}$ groups. Additionally, the peripheral hemolytic index was highly correlated with the hemolytic index $(r=0.656, p<0.001)$.

Conclusions: Our findings indicate that electrolytes reduce hemolysis by Candida species and a correlation exists between the peripheral hemolytic index and the translucent hemolytic index.

\section{Background}

Pathogenic fungi have become an increasing risk factor for people suffering from systemic disease and patients with impaired immune systems. Numerous fungi have been described, but only a small group, including Candida species, is pathogenic. Candida species can cause superficial mucosal infections and deadly systemic disease. In recent years, the number of Candida infections has increased dramatically and many have resulted in mortality [1-4].

Therefore, a better understanding of Candida species pathogenicity will undoubtedly be beneficial for the treatment of Candida infections. Although the epidemiology of Candida species has been thoroughly studied, the virulence is not well understood. Virulence factors, such as adherence, extracellular hydrolase production, hemolysis, phenotypic switching, and filamentation may all influence the pathogenesis of Candida species. A variety of virulence factors are expressed by Candida albicans for the adaptation to specific anatomical sites

\footnotetext{
*Correspondence: gzhcao@smu.edu.cn; jincaizhang@live.cn

${ }^{\dagger}$ Equal contributors

'Department of Microbiology, School of Public Health and Tropical Medicine, Southern Medical University, Guangzhou, China

${ }^{2}$ Department of Periodontology, Guangdong Provincial Stomatological

Hospital, Southern Medical University, Guangzhou, China

Full list of author information is available at the end of the article
}

[5]. As one of the most important virulence factors of $C$. albicans, secreted aspartate proteinases (Saps) have been studied fully [6], while its hemolytic activity is not well understood. Hemolytic activity is a potential virulence factor that helps to disseminate candidiasis and facilitate hyphal invasion [7]. The hemolytic activity of Candida is enhanced by growth on glucose-enriched blood agar, as first described by Manns et al. [8]. Candida albicans uses hemolysins to degrade hemoglobin and obtain elemental iron. Therefore, hemolysins are crucial virulence factors that help pathogens to survive and persist [8-10], but for Candida isolates, the features of hemolysins are poorly characterized [11].

A limited number of studies have explored the influence of specific ions on the hemolysins of Candida isolates. N. Yigit and Aktas [12] compared the use of different blood media on the hemolysins of Candida isolates and found that sheep blood Sabouraud dextrose agar is the most suitable for the study of the betahemolytic activity of Candida isolates, and Linares et al. [13] observed that $\mathrm{CaCl}_{2}$ affected the hemolytic activity. When $2.5 \% \mathrm{CaCl}_{2}$ was added to Sabouraud glucose agar supplemented with sheep blood, the hemolytic activity of C. dubliniensis was reduced and that of the C. albicans strains was stimulated. However, in the absence of 
$2.5 \% \mathrm{CaCl}_{2}$, the hemolytic activities of $C$. albicans and $C$. dubliniensis were not different.

Despite these previous studies, the effect of specific electrolytes on the hemolysins of Candida strains remains unclear. In particular, the relationship between common electrolytes and the hemolysins of Candida strains has not been explored. Although hemolysis may be effective at promoting the pathogenesis of Candida strains, additional studies of potential hemolytic factors are needed. This study was designed to test the influence of specific electrolytes on the hemolysins of Candida strains.

\section{Methods}

\section{Candida species}

Sixteen strains of C. glabrata, four C. albicans and one C. tropicalis recovered from clinical specimens of different patients at the Guangzhou Eighth People's Hospital and preserved in the laboratory were included in the study. Additionally, a single strain each of C. albicans (ATCC 90028) (American Type Culture Collection, Manassas, VA, USA), C. glabrata (ATCC 90030), C. krusei (ATCC 6258) and C. tropicalis (ATCC 13803) were included for data comparison purposes. The API 20C Aux Identification Kit (Bio Merieux SA, Lyon, France) and the "germ tube test" were used to identity all the isolates. Cultures were stored at $-79{ }^{\circ} \mathrm{C}$. Once recovered from the patients, they were kept on Sabouraud dextrose agar (Guangzhou Detgerm Microbiology Technology Co., Guangzhou, China), and stored at $4{ }^{\circ} \mathrm{C}$. The identification of each isolate was performed separately to guarantee their purities.

All the clinical isolates used in this study, which were not traceable to the donors, have been used previously and details of their isolation have been described [10, 14]. The hemolytic activity of every strain used in this study was determined twice in the absence of added electrolytes. The three electrolytes, $\mathrm{CaCl}_{2}, \mathrm{NaCl}$ and $\mathrm{KCl}$ (Aladdin Industrial Corporation, Shanghai, China), were added during the preparation of the plates.

\section{Preparation of plates with various electrolytes at different concentrations}

The control medium was prepared by adding $7 \mathrm{~mL}$ of fresh sheep blood (Hemostat, Dixon, CA, USA) to $100 \mathrm{~mL}$ of Sabouraud dextrose agar containing $3 \%$ glucose (final concentration, wt/vol; Guangzhou Detgerm Microbiology Technology Co., Guangzhou, China). The $\mathrm{pH}$ was $5.6 \pm 0.2$. $\mathrm{NaCl}$ or $\mathrm{KCl}$ was added to the above medium at concentrations of $1,2.5$ or $5 \%$ (wt/vol), while $\mathrm{CaCl}_{2}$ was added at concentrations of $0.5,1$ or $2.5 \%$ (wt/vol).

\section{Quantification of hemolytic activity}

The method of Luo et al. [14] was used to assess hemolytic activity. Isolates were cultured in Sabouraud dextrose agar at $37^{\circ} \mathrm{C}$ for $24 \mathrm{~h}$. Then, the cultures were collected and washed with sterile saline and a yeast suspension was prepared from an inoculum of $1 \times 10^{8}$ cells $/ \mathrm{mL}$ by hemocytometric counts. Ten microliters was spotted on each plate. Plates were cultured at $37^{\circ} \mathrm{C}$ in a $5 \% \mathrm{CO}_{2}$ atm for $48 \mathrm{~h}$. A translucent halo around the inoculum indicated the presence of hemolytic activity. The ratio calculated by dividing the total diameter of the colony plus the translucent halo by the diameter of the colony was defined as the hemolytic index (Hi), which represented the intensity of hemolysin production. Moreover, a dark ring was observed at the periphery of the distinctive translucent halo on every plate. The ratio calculated by dividing the diameter of the peripheral dark ring, the translucent halo and the colony by the diameter of the colony was defined as the peripheral hemolytic index $(\mathrm{Hp})$, which represented the intensity of the peripheral hemolysin production.

The relative change of hemolytic activity for a specific electrolyte at a specific concentration compared with the control group was calculated as follows: the relative change of the test culture hemolytic index $=$ (test culture hemolytic index-hemolytic index of the control culture)/hemolytic index of the control culture.

\section{Statistical analysis}

All experiments were performed in duplicate. All data were assessed for homogeneity of variance using the Levene index and expressed as the mean \pm standard deviation. Hemolytic activities in the presence of a specific electrolyte at different concentrations were analyzed by the repeated measures test. A one-way analysis of variance (ANOVA) was used to assess the degree of change in hemolytic activity for each electrolyte at different concentrations. If there was heterogeneity of variance, the rank-sum test was used instead of one-way ANOVA. Bivariate correlation analysis was applied to calculate the relationship between $\mathrm{Hi}$ and $\mathrm{Hp}$. All statistical analyses were carried out using SPSS 13.0 (SPSS Inc., Chicago, IL, USA) and $p<0.05$ was considered to be statistically significant, while $p<0.01$ was considered highly statistically significant.

\section{Results}

Three time points, 24,48 , and $72 \mathrm{~h}$, were selected for analysis. At $24 \mathrm{~h}$ post-inoculation, only partial hemolysis was observed. At $72 \mathrm{~h}$ post-inoculation, the zone of hemolysis in some plates became opaque. This phenomenon became more common after prolonged incubation. Therefore, the 48-h time point was chosen for comparison, which was consistent with previous studies [12, 13, 15]. All the clinical strains and the standard strains showed hemolytic activity in the presence of added electrolytes under all tested conditions. 
Differences in the hemolytic activity of each electrolyte at different concentrations

\section{Effect of calcium chloride on the hemolytic activity of}

\section{Candida isolates}

To determine whether $\mathrm{CaCl}_{2}$ will affect the hemolytic activity of Candida isolates, we used plates supplemented with $\mathrm{CaCl}_{2}$ at $0.5,1$, or $2.5 \%$ (wt/vol). Any decrease in the observed hemolytic activity was compared with the control plates. Comparison of the hemolytic indices among the groups treated with $0.5 \% \mathrm{CaCl}_{2}(\mathrm{Hi}=2.247 \pm 0.079), 1 \%$ $\mathrm{CaCl}_{2}(\mathrm{Hi}=2.013 \pm 0.092), 2.5 \% \mathrm{CaCl}_{2}(\mathrm{Hi}=2.150 \pm 0.066)$ and the control $(\mathrm{Hi}=2.749 \pm 0.103)$ reached statistical significance $(p<0.001)$. A typical result is shown in Fig. 1 .

\section{Effect of sodium chloride on the hemolytic activity of Candida isolates}

To determine whether $\mathrm{NaCl}$ will affect the hemolytic activity of Candida isolates, plates including $\mathrm{NaCl}$ at 1, 2.5 or $5 \%$ (wt/vol) were used. Any decrease in the observed hemolytic activity was compared with the control plates. Comparison of the hemolytic indices among the groups treated with $1 \% \mathrm{NaCl}(\mathrm{Hi}=2.158 \pm 0.078), 2.5 \% \mathrm{NaCl}$ $(\mathrm{Hi}=1.724 \pm 0.077), 5 \% \mathrm{NaCl}(\mathrm{Hi}=1.746 \pm 0.102)$ and the control $(\mathrm{Hi}=2.839 \pm 0.156)$ reached statistical significance $(p<0.001)$. A typical result is shown in Fig. 2.

\section{Effect of potassium chloride on the hemolytic activity of Candida isolates}

To determine whether $\mathrm{KCl}$ will affect the hemolytic activity of Candida isolates, plates containing $\mathrm{KCl}$ at 1, 2.5 or $5 \%$ (wt/vol) were used. Any decrease in the observed hemolytic activity observed was compared with the control plates. Comparison of the hemolytic indices among the groups treated with $1 \% \mathrm{KCl}(\mathrm{Hi}=1.867 \pm 0.077), 2.5 \% \mathrm{KCl}(\mathrm{Hi}=$ $1.642 \pm 0.079), 5 \% \mathrm{KCl}(\mathrm{Hi}=1.707 \pm 0.089)$ and the control $(\mathrm{Hi}=2.839 \pm 0.156)$ reached statistical significance $(p<$ 0.001). A typical result is shown in Fig. 3.

\section{Relative change of hemolytic activity in response to} different concentrations of specific electrolytes

The relative changes in hemolytic activity for all three electrolytes at different concentrations were calculated

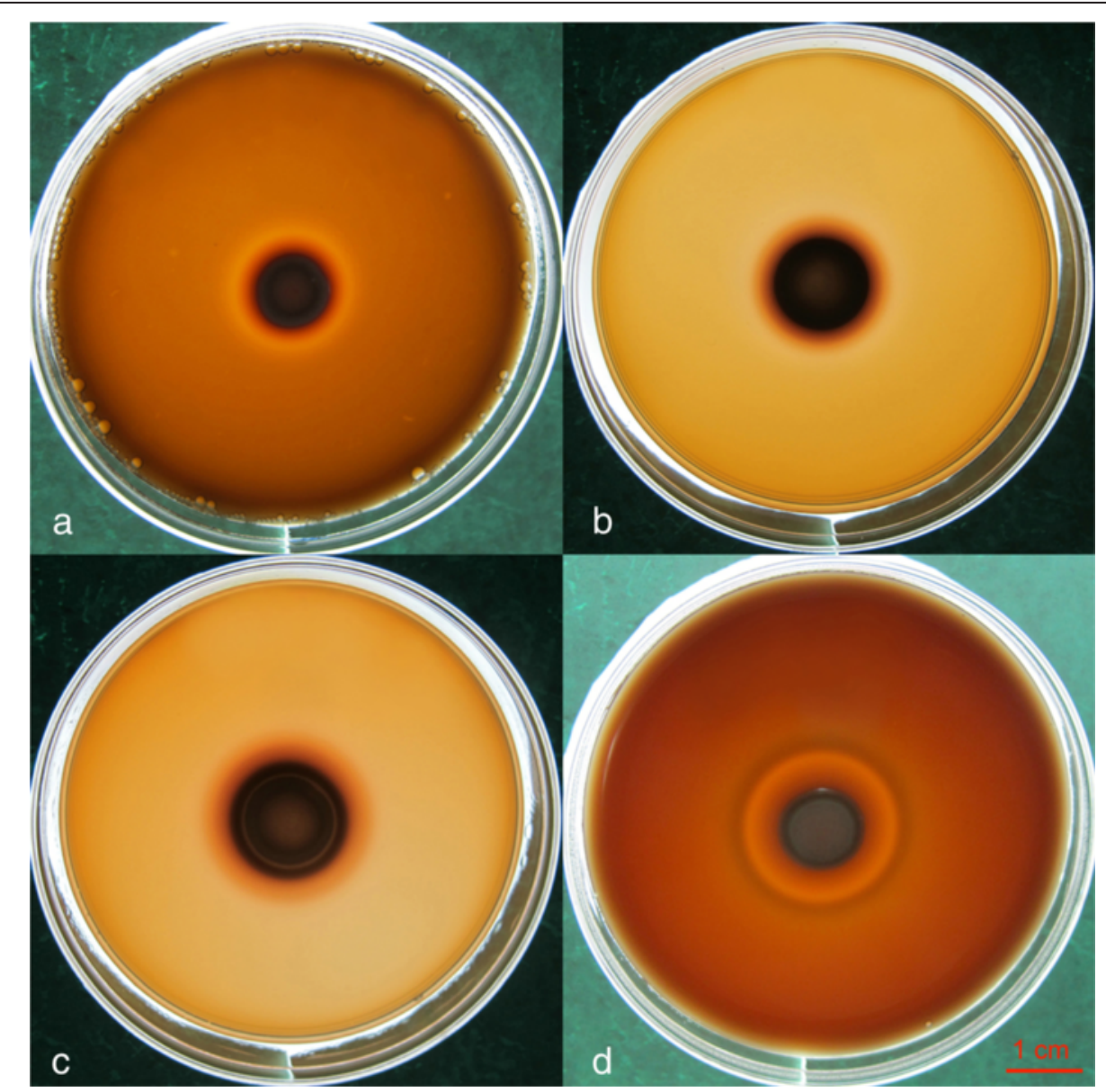

Fig. 1 Hemolysis caused by C. tropicalis (ATCC 13803) on plates containing different concentrations of $\mathrm{CaCl}_{2}$. a $0.5 \% \mathrm{CaCl}_{2}$, b $1 \% \mathrm{CaCl}_{2}$, c $2.5 \%$ $\mathrm{CaCl}_{2}$, and $\mathbf{d}$ control 


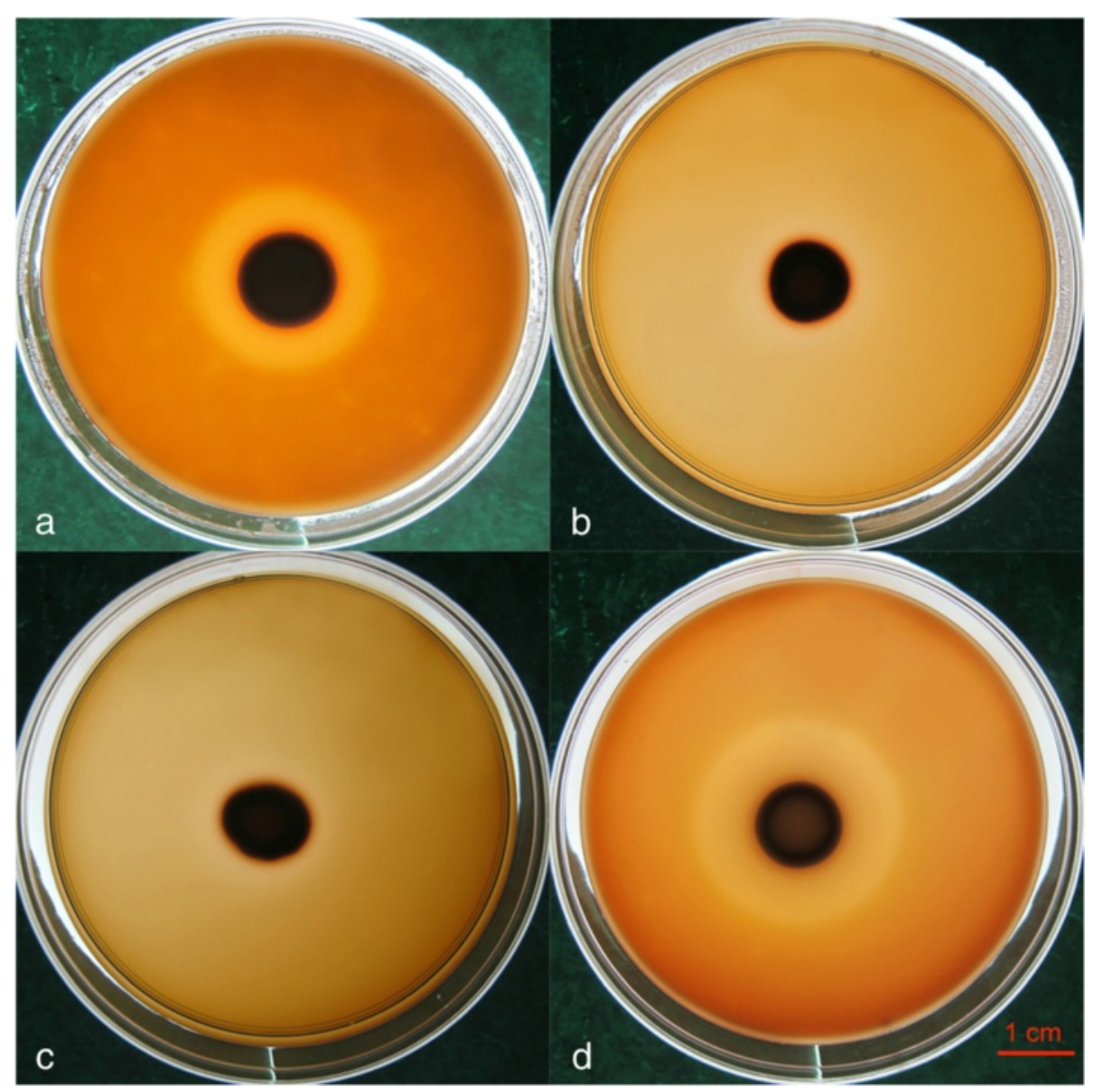

Fig. 2 Hemolysis caused by a C. glabrata clinical strain on plates containing different concentrations of $\mathrm{NaCl}$. a $1 \% \mathrm{NaCl}$, b $2.5 \% \mathrm{NaCl}$, c $5 \%$ $\mathrm{NaCl}$, and $\mathbf{d}$ control

according to the method described above. Significant differences existed between different concentrations for the $\mathrm{NaCl}$ group $(F=13.967, p<0.001)$, while no significant differences existed between the different concentrations for the $\mathrm{CaCl}_{2}$ group $(F=2.727, p=0.074)$. The Levene index of the $\mathrm{KCl}$ group was 0.001 ; therefore, the rank-sum test was used. The result of the $\mathrm{KCl}$ group was $\chi^{2}=3.734, p=0.155$. For multiple comparisons among different concentrations, in accordance with a significance level of $\alpha=0.05 / 3$, no significant difference existed in the $\mathrm{KCl}$ group. The specific values are shown in Table 1.

\section{Relationship between $\mathrm{Hp}$ and $\mathrm{Hi}$}

After integrating all the data from the $\mathrm{CaCl}_{2}$ group, the $\mathrm{NaCl}$ group and the $\mathrm{KCl}$ group, the coefficient for the correlation between the peripheral hemolytic index and the hemolytic index was $0.656(p<0.001)$.

\section{Discussion}

A previous study showed that the hemolytic activity of C. dubliniensis and C. albicans was affected by $2.5 \%$
$\mathrm{CaCl}_{2}$ [13]. Therefore, we selected bracketing concentrations of $1,2.5$, and $5 \%$ for studying the electrolytes $\mathrm{NaCl}$ and $\mathrm{KCl}$. Because the addition of $5 \% \mathrm{CaCl}_{2}$ to the agar prevented the agar from solidifying, the concentrations chosen for the $\mathrm{CaCl}_{2}$ group were $0.5,1$, and $2.5 \%$.

Many factors can influence hemolytic activity, such as temperature [16], glucose [14], certain ions, including $\mathrm{Fe}^{3+}$ [17], certain compounds (ethanol, $n$-butanol, or $n-$ pentanol vapor) [18]' or the species that is the source of the blood [12]. Franca et al. [19] analyzed the hemolytic activities of Candida strains that were obtained from the same site and showed that C. tropicalis blood isolates had significantly higher activity. However, when they compared the hemolytic activities of Candida strains from different sites, the results showed that the hemolytic activity of C. parapsilosis isolates from tracheal secretions was higher. In addition, different batches of media, the thickness of the media, and the vitality of the strain can all affect the hemolytic activity of Candida species. However, some factors have no impact on the hemolytic activity. Favero [15] indicated that incubation in a normal environment or 


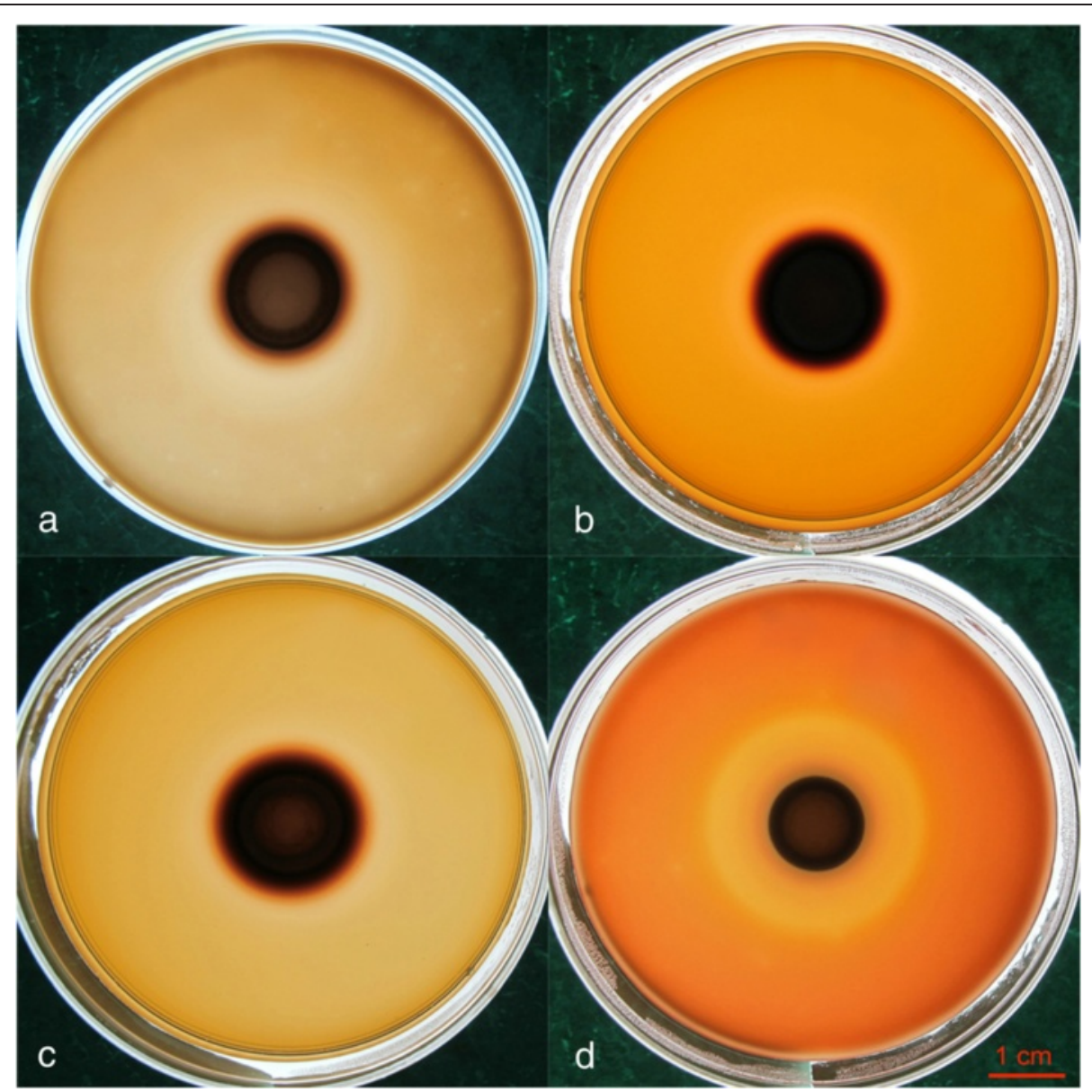

Fig. 3 Hemolysis caused by a C. albicans clinical strain on plates containing different concentrations of $\mathrm{KCl}$. a $1 \% \mathrm{KCl}$, b $2.5 \% \mathrm{KCl}$, c $5 \% \mathrm{KCl}$, and $\mathbf{d}$ control

increased $\mathrm{CO}_{2}$ did not have any effect on hemolysis by $C$. tropicalis, nor was the hemolytic activity of $C$. tropicalis suppressed by heat treatment $\left(100{ }^{\circ} \mathrm{C}\right)$ or by supplementation with pepstatin A. However, for beta-hemolytic Streptococci, an increased $\mathrm{CO}_{2}$ atm (5-10\%) promoted hemolysis [20]. Thus, an increased $\mathrm{CO}_{2}$ environment may also influence the hemolytic activity of Candida in a distinct manner [15].

Table 1 Relative change of hemolytic activity in response to different concentrations of specific electrolytes

\begin{tabular}{|c|c|c|c|c|c|c|}
\hline \multirow{3}{*}{\multicolumn{2}{|c|}{$\begin{array}{l}\text { Levene index } \\
p\end{array}$}} & \multicolumn{2}{|c|}{ Analysis of variance } & \multicolumn{3}{|c|}{ Pairwise comparisons } \\
\hline & & \multirow[t]{2}{*}{$\mathrm{F}$} & \multirow[t]{2}{*}{$p$} & \multirow{2}{*}{$\begin{array}{l}\text { A vs. B } \\
p\end{array}$} & \multirow{2}{*}{$\begin{array}{l}\text { A vs. C } \\
p\end{array}$} & \multirow{2}{*}{$\begin{array}{l}\text { B vs. C } \\
p\end{array}$} \\
\hline & & & & & & \\
\hline $\mathrm{CaCl}_{2}$ & 0.438 & 2.727 & 0.074 & 0.082 & 1.000 & 0.319 \\
\hline $\mathrm{NaCl}$ & 0.354 & 13.967 & $<0.001$ & $<0.001$ & $<0.001$ & 1.000 \\
\hline $\mathrm{KCl}$ & 0.001 & 3.642 & 0.033 & 0.040 & 0.145 & 1.000 \\
\hline
\end{tabular}

$A, B$, and $C$ each represent the concentration of an electrolyte and are arranged from lowest to highest. In the $\mathrm{CaCl}_{2}$ group, A represents $0.5 \% \mathrm{CaCl}_{2}$, B represents $1 \% \mathrm{CaCl}_{2}$ and $\mathrm{C}$ represents $2.5 \% \mathrm{CaCl}_{2}$, while in the $\mathrm{NaCl}$ and $\mathrm{KCl}$ groups, A represents $1 \%, \mathrm{~B}$ represents $2.5 \%$ and $\mathrm{C}$ represents $5 \%$ of each respective electrolyte
Before we tested the effects of electrolytes on Candida species hemolysis, the hemolytic activity of every strain was evaluated twice in the absence of added electrolytes. All the strains showed hemolytic activity. After adding the electrolytes, every strain still showed positive hemolysis. However, our results suggested that when grown in $\mathrm{CaCl}_{2}$, $\mathrm{NaCl}$, or $\mathrm{KCl}$, the hemolytic activity of Candida isolates was reduced. In patients, the levels of these electrolytes depend on a myriad of factors. The relative balance of electrolytes is important to the health and function of the whole body, and electrolyte disorders are common in cardiovascular patients [21], in older community subjects [22], and in diabetic outpatients, even if their renal function is normal [23]. Additionally, patients with malignancies commonly experience serum electrolyte abnormalities, including hyponatremia, hypokalemia, hyperkalemia, hypophosphatemia, and hypercalcemia [24].

Electrolyte disorders are complicated to analyze in vivo, and it is impractical to simulate their complexity in vitro. In local environments, such as oral mucosa or vaginal mucosa, exogenous substances, such as food, drugs and rinses, can influence the electrolyte balances. The use of toothpaste 
containing calcium bicarbonate may be helpful for the prevention of oral candidiasis. Other strategies such as rinses containing certain electrolytes may be useful for the prevention of vaginal candidiasis. Whether an increase in the level of a specific electrolyte in a local environment will have an impact on the hemolytic activity of Candida isolates is currently unknown. Our results show that hemolytic activity in the presence of the three selected electrolytes was decreased compared with the control group. The relative change in hemolytic activity for all three electrolytes at different concentrations was also determined. No significant differences existed in the hemolytic activity of Candida between different concentrations of $\mathrm{CaCl}_{2}$ and $\mathrm{KCl}$. However, significant differences were evident between the relative hemolytic activities in the presence of $1 \% \mathrm{NaCl}$ and $2.5 \% \mathrm{NaCl}$, and $1 \% \mathrm{NaCl}$ and $5 \% \mathrm{NaCl}$. A concentration of $1 \% \mathrm{NaCl}$ is slightly higher than the normal blood concentration $(0.9 \% \mathrm{NaCl})$ indicating that the hemolytic activity of Candida species under conditions of hypernatremia is decreased, which may be useful for the clinical management of patients with electrolyte disturbances.

To date, there are few studies of ions and their influence on hemolytic activity. $\mathrm{Fe}^{3+}$ released from orthodontic appliances could reduce hemolytic activity [17]. Many environmental factors, such as extracellular $\mathrm{pH}$ and the concentrations of alkali metal cations can affect the virulence of Candida isolates. Generally speaking, Candida species can grow at relatively high $\mathrm{NaCl}$ concentrations [25], although salt has a negative impact on some virulence characteristics [26]. A relationship exists between the formation of $C$. albicans hyphae and the intracellular concentration of potassium [27]. Yeast species have transport systems to maintain homeostasis in the presence of a high ratio of potassium and sodium concentrations [28, 29]. Most yeasts have only one type of antiporter to efficiently transport both potassium and sodium cations from the cells $[29,30]$. The $\mathrm{Na}^{+} / \mathrm{H}^{+}$antiporter is an important transport system whose activity can influence the tolerance of Candida strains to high external concentrations of alkali metal cations [31].

A previous study showed that $\mathrm{Ca}^{2+}$ can inhibit the bactericidal effect of human lactoferrin [32]. Although the reason for this inhibitory effect on candidacidal activity remains unclear, it could be a result of many factors [33]. Researchers previously reported the extracellular cation concentration influenced the bactericidal effect of lactoferrin [34] and assumed that human lactoferrin interacted with the $C$. albicans cytoplasmic membrane in a manner that made it candidacidal [35]. More detailed studies investigating the mechanisms for the decreased hemolytic activity observed in the presence of electrolytes are required.

In this study, we tested the hemolytic activity of 25 Candida isolates. At $48 \mathrm{~h}$ post-inoculation, two different types of hemolysis were observed surrounding the yeast (Fig. 4). The first type produced a highly translucent outer ring, and the second type produced a dark peripheral halo, hence, the terms hemolytic index and peripheral hemolytic index were used to describe these two types of hemolysis, respectively. The results conclusively indicated that there was a strong correlation between $\mathrm{Hi}$ and Hp. Future work may include an in-depth study of Candida hemolysis using chemical analysis to determine the differences between peripheral hemolysis and translucent hemolysis by investigating their chemical composition, properties, structure, and functions.

\section{Conclusions}

Our clinically relevant findings indicate that different electrolytes can modulate the activity of hemolysins, potential virulence factors found in Candida species. All the electrolytes tested in this study produced a decrease

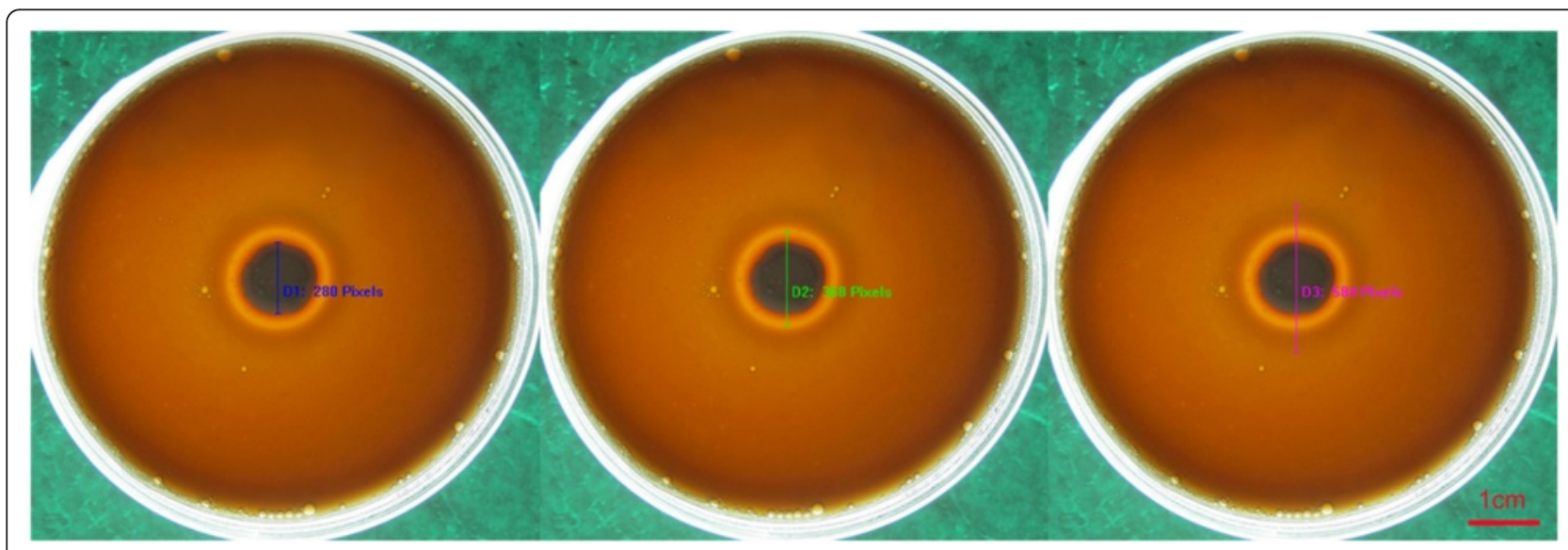

Fig. 4 Two different types of hemolytic activity observed around the yeast. The terms hemolytic index (Hi=D2/D1) and peripheral hemolytic index $(\mathrm{Hp}=\mathrm{D3} / \mathrm{D} 1)$ were used to indicate these two different forms of hemolysis. The first term denotes a highly translucent ring and the second term describes a peripheral dark halo 
in the hemolysis observed by the Candida species. To our knowledge, this is the first time that a correlation between the peripheral hemolytic index and the translucent hemolytic index has been identified. The results of this study expand our knowledge of factors affecting Candida hemolysis.

\section{Abbreviations}

Saps: Secreted aspartate proteinase; ATCC: American Type Culture Collection; $\mathrm{Hi}$ : The hemolytic index; Hp: The peripheral hemolytic index; ANOVA: Analysis of variance.

\section{Competing interests}

The authors declare that they have no competing interests.

\section{Authors' contributions}

LW was responsible for carrying out the research and writing and revising the manuscript. GL participated directly in the interpretation of data and also contributed to the acquisition of the materials used in the present research. $\mathrm{HBL}$ significantly contributed to the execution of the methodology and acquisition and configuration of the images. DYX edited the paper. HC and JCZ were responsible for the study design and supervision of the manuscript. All authors read and approved the final manuscript.

\section{Acknowledgements}

This research was supported by grants from the National Natural Science Foundation of China (Grant 81371151) to J. Zhang, the Natural Science Fund of Guangdong province of China (Grant 2014A030310146) to H. Lu, and the National Natural Science Foundation of China (Grant 81271159/ H1405) to G. Luo.

\section{Author details}

'Department of Microbiology, School of Public Health and Tropical Medicine, Southern Medical University, Guangzhou, China. ${ }^{2}$ Department of Periodontology, Guangdong Provincial Stomatological Hospital, Southern Medical University, Guangzhou, China. ${ }^{3}$ Key Laboratory of Oral Medicine,Guangzhou Institute of Oral Disease,Stomatology Hospital of Guangzhou Medical University, Guangzhou 510140, China.

\section{Received: 9 December 2014 Accepted: 6 August 2015}

Published online: 22 August 2015

\section{References}

1. Comert F, Kulah C, Aktas E, Eroglu O, Ozlu N. Identification of Candida species isolated from patients in intensive care unit and in vitro susceptibility to fluconazole for a 3-year period. Mycoses. 2007:50(1):52-7.

2. Caggiano G, latta R, Laneve A, Manca F, Montagna MT. Observational study on candidaemia at a university hospital in southern Italy from 1998 to 2004. Mycoses. 2008;51(2):123-8.

3. Malafiej E, Adamiec AC, Tworzyanska U. Microbial profile and drug resistance of Candida strains isolated from the blood of children: an 11-year study. Mycoses. 2009;52(2):149-53.

4. Ahmad A, Khan AU. Prevalence of Candida species and potential risk factors for vulvovaginal candidiasis in Aligarh, India. Eur J Obstet Gynecol Reprod Biol. 2009:144(1):68-71.

5. Calderone RA, Fonzi WA. Virulence factors of Candida albicans. Trends Microbiol. 2001:9(7):327-35

6. Schaller M, Borelli C, Korting HC, Hube B. Hydrolytic enzymes as virulence factors of Candida albicans. Mycoses. 2005:48(6):365-77.

7. Odds FC. Candida and Candidosis. 2nd ed. London, UK: Bailliere Tindall; 1988

8. Manns JM, Mosser DM, Buckley HR. Production of a hemolytic factor by Candida albicans. Infect Immun. 1994:62(11):5154-6.

9. Watanabe T, Takano M, Murakami M, Tanaka H, Matsuhisa A, Nakao N, et al. Characterization of a haemolytic factor from Candida albicans. Microbiology. 1999;145(Pt 3):689-94.

10. Luo G, Samaranayake LP, Cheung BP, Tang G. Reverse transcriptase polymerase chain reaction (RT-PCR) detection of HLP gene expression in Candida glabrata and its possible role in in vitro haemolysin production. Apmis. 2004;112(4-5):283-90.
11. Silva S, Negri M, Henriques M, Oliveira R, Williams DW, Azeredo J. Candida glabrata, Candida parapsilosis and Candida tropicalis: biology, epidemiology, pathogenicity and antifungal resistance. Fems Microbiol Rev. 2012;36(2):288-305.

12. Yigit $\mathrm{N}$, Aktas E. Comparison of the efficacy of different blood medium in determining the hemolytic activity of Candida species. Journal de Mycologie Médicale. 2009;19:110-5.

13. Linares CE, de Loreto ES, Silveira CP, Pozzatti P, Scheid LA, Santurio JM, et al. Enzymatic and hemolytic activities of Candida dubliniensis strains. Rev Inst Med Trop Sao Paulo. 2007:49(4):203-6.

14. Luo G, Samaranayake LP, Yau JY. Candida species exhibit differential in vitro hemolytic activities. J Clin Microbiol. 2001;39(8):2971-4.

15. Favero D, Franca EJ, Furlaneto-Maia L, Quesada RM, Furlaneto MC. Production of haemolytic factor by clinical isolates of Candida tropicalis. Mycoses. 2011;54(6):e816-20.

16. Tanaka WT, Nakao N, Mikami T, Matsumoto T. Hemoglobin is utilized by Candida albicans in the hyphal form but not yeast form. Biochem Biophys Res Commun. 1997;232(2):350-3.

17. Ronsani MM, Mores RA, Meira TM, Trindade GA, Guariza FO, Tanaka OM, et al. Virulence modulation of Candida albicans biofilms by metal ions commonly released from orthodontic devices. Microb Pathog. 2011;51(6):421-5.

18. Shuster A, Osherov N, Rosenberg M. Alcohol-mediated haemolysis in yeast Yeast. 2004:21(16):1335-42

19. Franca E, Furlaneto-Maia L, Quesada RM, Favero D, Oliveira MT, Furlaneto MC. Haemolytic and proteinase activities in clinical isolates of Candida parapsilosis and Candida tropicalis with reference to the isolation anatomic site. Mycoses. 2011;54(4):e44-51.

20. Ruoff KL. Streptococcus. In: Murray PR, Baron EJ, Pfaller MA,Tenover FC, Yolken RH(eds), Manual of Clinical Microbiology. Washington, DC: American Society for Microbiology, 1995;299-305

21. Hinkle C. Electrolyte disorders in the cardiac patient. Crit Care Nurs Clin North Am. 2011;23(4):635-43

22. Liamis G, Rodenburg EM, Hofman A, Zietse R, Stricker BH, Hoorn EJ. Electrolyte disorders in community subjects: prevalence and risk factors. Am J Med. 2013;126(3):256-63.

23. Sotirakopoulos N, Kalogiannidou I, Tersi M, Armentzioiou K, Sivridis D, Mavromatidis K. Acid-base and electrolyte disorders in patients with diabetes mellitus. Saudi J Kidney Dis Transpl. 2012;23(1):58-62.

24. Rosner MH, Dalkin AC. Electrolyte disorders associated with cancer. Adv Chronic Kidney Dis. 2014;21(1):7-17.

25. Alves SH, Milan EP, de Laet SP, Oliveira LO, Santurio JM, Colombo AL. Hypertonic sabouraud broth as a simple and powerful test for Candida dubliniensis screening. Diagn Microbiol Infect Dis. 2002:43(1):85-6.

26. Hermann P, Forgacs K, Gal E, Lenkey B, Nagy G, Rozgonyi F. Effects of alkali metal ions on some virulence traits of Candida albicans. Folia Microbiol (Praha). 2003;48(2):173-6.

27. Watanabe $\mathrm{H}$, Azuma M, Igarashi $\mathrm{K}$, Ooshima H. Relationship between cell morphology and intracellular potassium concentration in Candida albicans. J Antibiot (Tokyo). 2006;59(5):281-7.

28. Rodriguez-Navarro A. Potassium transport in fungi and plants. Biochim Biophys Acta. 2000;1469(1):1-30.

29. Kinclova O, Ramos J, Potier S, Sychrova H. Functional study of the Saccharomyces cerevisiae Nha1p C-terminus. Mol Microbiol. 2001;40(3):656-68.

30. Velkova K, Sychrova H. The Debaryomyces hansenii NHA1 gene encodes a plasma membrane alkali-metal-cation antiporter with broad substrate specificity. Gene. 2006:369:27-34.

31. Krauke $Y$, Sychrova $\mathrm{H}$. Functional comparison of plasma-membrane $\mathrm{Na}^{+} / \mathrm{H}^{+}$ antiporters from two pathogenic Candida species. BMC Microbiol. 2008;8:80.

32. Ellison RR, LaForce FM, Giehl TJ, Boose DS, Dunn BE. Lactoferrin and transferrin damage of the gram-negative outer membrane is modulated by $\mathrm{Ca}^{2+}$ and $\mathrm{Mg}^{2+}$. J Gen Microbiol. 1990;136(7):1437-46.

33. Bennett RM, Bagby GC, Davis J. Calcium-dependent polymerization of lactoferrin. Biochem Biophys Res Commun. 1981;101(1):88-95.

34. Viejo-Diaz M, Andres MT, Perez-Gil J, Sanchez M, Fierro JF. Potassium efflux induced by a new lactoferrin-derived peptide mimicking the effect of native human lactoferrin on the bacterial cytoplasmic membrane. Biochemistry (Mosc). 2003:68(2):217-27.

35. Viejo-Diaz M, Andres MT, Fierro JF. Modulation of in vitro fungicidal activity of human lactoferrin against Candida albicans by extracellular cation concentration and target cell metabolic activity. Antimicrob Agents Chemother. 2004:48(4):1242-8. 